\title{
The neocortical column
}

\author{
Javier DeFelipe ${ }^{1 *}$, Henry Markram ${ }^{2}$ and Kathleen S. Rockland ${ }^{3}$ \\ Instituto Cajal, Consejo Superior de Investigaciones Científicas, Centro de Tecnología Biomédica, Universidad Politécnica de Madrid, Madrid, Spain \\ 2 Ecole Polytechnique Federale de Lausanne, Lausanne, Switzerland \\ 3 Picower Institute for Learning and Memory, Massachusetts Institute of Technology, Cambridge, MA, USA \\ *Correspondence: defelipe@cajal.csic.es
}

Edited by:

Idan Segev, The Hebrew University of Jerusalem, Israel

Reviewed by:

Idan Segev, The Hebrew University of Jerusalem, Israel

In the middle of the twentieth century, Rafael Lorente de Nó (1902-1990) introduced the fundamental concept of the "elementary cortical unit of operation," proposing that the cerebral cortex is formed of small cylinders containing vertical chains of neurons (Lorente de Nó, 1933, 1938). On the basis of this idea, the hypothesis was later developed of the columnar organization of the cerebral cortex, primarily following the physiological and anatomical studies of Vernon Mountcastle, David Hubel, Torsten Wiesel, János Szentágothai, Ted Jones, and Pasko Rakic (for a review of these early studies, see Mountcastle, 1998). The columnar organization hypothesis is currently the most widely adopted to explain the cortical processing of information, making its study of potential interest to any researcher interested in this tissue, both in a healthy and pathological state. However, it is frequently remarked that the nomenclature surrounding this hypothesis often generates problems, as the term "Column" is used freely and promiscuously to refer to multiple, distinguishable entities, such as cellular or dendritic minicolumns or afferent macrocolumns, with respective diameters of $<50$ and $200-500 \mu \mathrm{m}$. Another problem is the degree to which classical criteria may need to be modified (shared response properties, shared input, and common output) and if so, how. Moreover, similar problems arise when we consider the need to define area-specific and species-specific variations. Finally, and what is more an ultimate goal than a problem, it is still necessary to achieve a better fundamental understanding of what columns are and how they are used in cortical processes. Accordingly, it is now very important to translate recent technical advances and new findings in the neurosciences into practical applications for neuroscientists, clinicians, and for those interested in comparative anatomy and brain evolution.

This volume contains nine articles that are intended to provide a summary of our current thoughts on the neocortical column. Three of them (those written by Rockland, da Costa and Martin, and by Rinkus) deal with the nomenclature and more theoretical issues, while the remaining articles include studies on comparative (Raghanti et al.) and developmental (Costa and Hedin-Pereira) aspects, as well as on the normal and altered cortical organization of these columns (Innocenti and Vercelli, Smit-Rigter et al., and Thomson). Finally, we have included a commentary on the analytical and quantitative tools that are currently available to define the diverse morphological patterns and functional parameters that characterize neurons (Losa et al.). The content of each of these articles is briefly summarized in more detail below.
The first article, by Rockland, mainly deals with the deceptively simple question "what is a column?" identifying five points for further discussion and re-evaluation: that anatomical columns are not solid structures; that they are part of locally interdigitated systems; that any delimited column also participates in a widely distributed network; that columns are not an obligatory cortical feature; and that columns (as "modules") occur widely in the brain even in non-cortical structures.

In the second article, da Costa and Martin describe the historical origins of the concept of the cortical column and the struggle that the pioneers faced to define its architecture. They suggest that within the concept of a "canonical circuit," we may find the means to reconcile the structure of the neocortex with its functional architecture. They propose that the concept of canonical microcircuit respects our understanding of the connectivity in the neocortex, and that the cortical column, as proposed, is sufficiently flexible to transiently adapt the architecture of its network in order to perform the required computations.

The third article by Rinkus is a hypothetical approach to examine the function of columns. The author proposes that the minicolumn has a generic functionality that only becomes clear when seen in the context of the higher-level functional unit: the macrocolumn. He proposes that a macrocolumn's function is to store sparsely distributed representations of its inputs and to recognize those inputs. Moreover, he claims that the generic function of the minicolumn is to enforce macrocolumnar code sparseness.

The fourth article is by Raghanti et al. and it is a review of the differences among species in minicolumns and GABAergic interneurons, discussing the possible implications for signaling between and within minicolumns. Furthermore, the authors discuss how abnormalities of minicolumn disposition and those of inhibitory interneurons might be associated with neuropathological processes, such as Alzheimer's disease, autism, and schizophrenia. Specifically explored is the possibility that the phylogenetic variability in the calcium-binding proteins expressed by distinct interneuron subtypes is directly related to differences in minicolumn morphology among species, as well as the possibility that this phenomenon might contribute to neuropathological susceptibility in humans.

In the fifth article, Costa and Hedin-Pereira address the relationships between cell lineage in the developing cerebral cortex and columnar organization. The authors describe cell lineage experiments that use replication-incompetent retroviral vectors to show that the progeny of a single neuroepithelial/radial glial cell in the dorsal telencephalon become organized into discrete radial clus- 
ters of excitatory neurons. These siblings have a higher propensity to develop chemical synapses with one another rather than with neighboring unrelated neurons. The authors discuss the possibility that the lineage of single neuroepithelial/radial glia cells could contribute to the columnar organization of the neocortex by generating radial columns of interconnected sibling neurons.

The sixth article by Innocenti and Vercelli reviews the proposal that the bundles of apical dendrites from pyramidal neurons belong to neurons projecting their axons to specific targets. The authors suggest that another structural and computational unit of the cerebral cortex is the cortical output unit. This output unit is an assembly of bundles of apical dendrites and their parent cell bodies, including each of the outputs to distant cortical or subcortical structures of a given cortical locus (area or part of an area).

The seventh article by Smit-Rigter et al. deals with the alterations in apical dendrite bundling in the somatosensory cortex of 5-HT3A receptor knockout mice. Using microtubule associated protein-2 immunostaining to visualize the apical dendrites of pyramidal neurons, the authors compare the apical dendritic bundles of wildtype and 5-HT3A receptor knockout mice. In the 5-HT3A receptor knockout mice, the surface of the dendritic bundle was larger than in the wild-type mice, while the number and distribution of reelinsecreting Cajal-Retzius cells was similar in both phenotypes. Along with the previously observed differences in the dendritic complexity of cortical layer $2 / 3$ pyramidal neurons and in the cortical reelin levels, the authors propose that the 5-HT3 receptor fulfills an important role in determining the spatial organization of cortical connectivity in the mouse somatosensory cortex.

In the eighth article, Thomson reviews aspects of layer VI and after briefly summarizing the development of this layer, describes and compares the major pyramidal cell classes found in layer VI. The connections made and received by these different classes of neurons are then discussed, as are the possible functional consequences of these connections, with particular reference to the shaping of physiological responses in the visual cortex and thalamus. Inhibition in layer VI is discussed where appropriate. Many types of interneurons can be found in each cortical layer and layer VI is no exception, although the functions of each type of interneuron remain to be elucidated.

The ninth article is by Losa et al. and it addresses the issues raised in an interesting article by Romand et al. (2011) on the morphological development of thick-tufted layer $V$ pyramidal cells in the rat somatosensory cortex. These pyramidal neurons are key elements of the columnar organization and Romand et al. used 3-D model neurons, reconstructed from biocytin-labeled cells, to study the principles that govern the dendritic and axonal arborization of these neurons. The methods used by Romand et al. are those employed commonly in many laboratories to analyze the neuronal components of the column and therefore, this article is of general interest to any researcher interested in column organization. However, the conclusions reached in their article are criticized by Losa et al. on the basis of the analytical procedure applied. According to Losa et al. the morphometric approaches used by Romand et al. (and as a consequence those used in most studies on cortical neurons) are usually termed conventional, as they are based on single scale measuring. Such an approach may be well suited to evaluate biological objects assumed to be, or arbitrarily approximated to, regular Euclidean structures, yet it is inappropriate to quantitatively describe the morphology of thick-tufted layer V pyramidal cells, which are characterized by complex functional properties and irregular morphological features. Thus, Losa et al. propose that an objective estimation can only be reached by applying the principles and rules of Fractal geometry. Nevertheless, Losa et al. conclude "that fractal and conventional morphometric approaches, built up on distinct epistemological principles, may set the understanding of the biologic reality at a different level. The former describes the morphological complexity within an experimental interval of observation scales that obviously encompasses the Euclidean dimension, while the latter proceeds at a primary level, i.e., by reducing cellular shapes and tissue structures to monotone elements which could be described by means of deterministic rules. Nevertheless, fractal and conventional morphometry may represent complementary analytical/quantitative tools to elucidate the diversity of morphological patterns and functional parameters which characterize neural cells and brain structures."

In summary, due to the general interest in the cortical columns, thousands of articles have been dedicated to this structure. Indeed, the article of Vernon Mountcastle describing the columnar organization of the cortex (Mountcastle, 1957) has been cited over 1462 times! Thus, while it is obvious that there are many issues and different points of view that have not been dealt with in the present e-book, we believe it fulfills our main intention of providing the reader with some interesting articles addressing different aspects and concepts associated with the organization of the neocortical column.

\section{REFERENCES}

Lorente de Nó, R. (1933). Studies on the structure of the cerebral cortex I. The area entorhinalis. J. Psychol. Neurol. 45, 381-438.

Lorente de Nó, R. (1938). "Architectonics and structure of the cerebral cortex," in Physiology of the Nervous System, ed. J. F. Fulton (New York: Oxford University Press), 291-330.

Mountcastle, V. B. (1957). Modality and topographic properties of single neurons of cat's somatic sensory cortex. J. Neurophysiol. 20, 408-434.

Mountcastle, V. B. (1998). Perceptual Neuroscience. The Cerebral Cortex. Cambridge: Harvard University Press.

Romand, S., Wang, Y., Toledo-Rodriguez, M., and Markram, H. (2011). Morphological development of thick-tufted layerV pyramidal cells in the rat somatosensory cortex. Front. Neuroanat. 5:5. doi: 10.3389/fnana.2011.00005

Received: 05 June 2012; accepted: 05 June 2012; published online: 26 June 2012. Citation: DeFelipe J, Markram H and Rockland KS (2012) The neocortical column. Front. Neuroanat. 6:22. doi: 10.3389/fnana.2012.00022

Copyright $(2012$ DeFelipe, Markram and Rockland. This is an open-access article distributed under the terms of the Creative Commons Attribution Non Commercial License, which permits non-commercial use, distribution, and reproduction in other forums, provided the original authors and source are credited. 